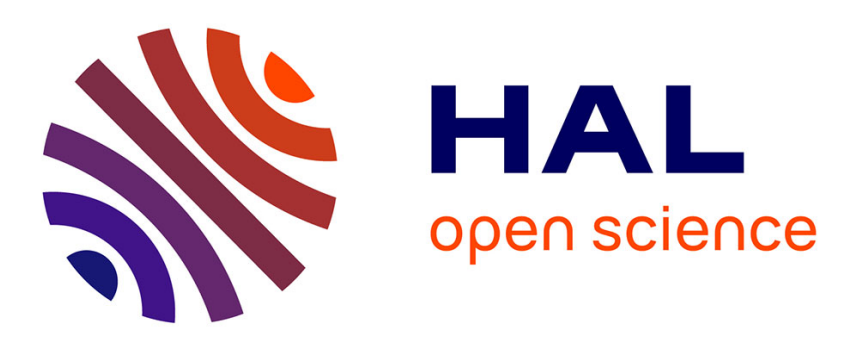

\title{
Stable and efficient Bloch-mode computational method for one-dimensional grating waveguides
}

Qing Cao, Philippe Lalanne, Jean-Paul Hugonin

\section{To cite this version:}

Qing Cao, Philippe Lalanne, Jean-Paul Hugonin. Stable and efficient Bloch-mode computational method for one-dimensional grating waveguides. Journal of the Optical Society of America. A Optics, Image Science, and Vision, 2002, 19 (2), pp.335-338. hal-00867916

\section{HAL Id: hal-00867916 \\ https://hal-iogs.archives-ouvertes.fr/hal-00867916}

Submitted on 30 Sep 2013

HAL is a multi-disciplinary open access archive for the deposit and dissemination of scientific research documents, whether they are published or not. The documents may come from teaching and research institutions in France or abroad, or from public or private research centers.
L'archive ouverte pluridisciplinaire HAL, est destinée au dépôt et à la diffusion de documents scientifiques de niveau recherche, publiés ou non, émanant des établissements d'enseignement et de recherche français ou étrangers, des laboratoires publics ou privés. 


\title{
Stable and efficient Bloch-mode computational method for one-dimensional grating waveguides
}

\author{
Qing Cao, Philippe Lalanne, and Jean-Paul Hugonin \\ Laboratoire Charles Fabry de l'Institut d'Optique, Centre National de la Recherche Scientifique, BP 147, \\ F-91403 Orsay Cedex, France
}

Received March 19, 2001; revised manuscript received June 28, 2001; accepted July 3, 2001

\begin{abstract}
We present a stable and efficient method for the Bloch-mode computation of one-dimensional grating waveguides. The approach uses the Fourier modal method and the $\mathbf{S}$-matrix algorithm to remove numerical instabilities. The use of perfectly matched layers provide a high accuracy. Numerical results obtained for different lamellar grating waveguides and for both TE and TM polarizations illustrate the performance of the approach. (C) 2002 Optical Society of America
\end{abstract}

OCIS codes: $050.1960,050.1950,130.2790,230.7390,350.5500$.

The rigorous coupled-wave analysis ${ }^{1,2}$ (RCWA) also called the Fourier modal method, is a well-established numerical technique for the numerical study of gratingdiffraction problems. It can efficiently solve almost all one-dimensional grating-stack diffraction problems by the combinative use of the $\mathbf{S}$ matrix or the $\mathbf{R}$ matrix. ${ }^{3} \mathrm{Re}-$ cently, by use of appropriate absorbers in the grating region, this powerful numerical tool was extended to the simulation of aperiodic diffraction problems, ${ }^{4}$ such as Bragg-grating waveguides, ${ }^{5}$ grating couplers, ${ }^{5}$ and photonic crystal waveguides. ${ }^{5-7}$ The extension was also applied to the Bloch-mode computation of one-dimensional grating waveguides. ${ }^{4}$

It is well established that the resonance anomalies of grating-waveguide filters, the coupling lengths of grating couplers, and the stop bands of Bragg waveguide mirrors and of surface-wave plasmons can be studied through the complex poles of the determinant of a scattering matrix that relates the diffracted and the incidence waves (see, for instance, Ref. 8 and references therein). In general, the computation of the poles (or, equivalently, the effective index $n_{\text {eff }}$ of the leaky mode) requires that one find the complex root of the determinant of the scattering matrix. This root search involves many iterative calculations of the determinant and often requires a good-guess value for the pole.

In contrast, the approach of Ref. 4 does not require any iteration; it straightforwardly computes the $n_{\text {eff }}$ value and the corresponding Bloch mode by solving for an eigenproblem. However, the transmission matrix ( $\mathbf{T}$ matrix) used in Ref. 4 for the eigenproblem is unstable when a large number $N$ of Fourier harmonics are retained for the computation or when large grating periods are considered. Obviously, this drawback greatly restricts the domain of application of the approach. For example, Bloch modes of highly conducting metal grating waveguides cannot be accurately computed. The purpose of this paper is to employ the $\mathbf{S}$ matrix to remove this limitation.
At the same time, we use perfectly-matched-layers (PML) techniques ${ }^{9,10}$ to enhance the computation accuracy.

Let us consider the lamellar grating waveguide shown in Fig. 1. The indices $n_{h}, n_{c}$, and $n_{s}$ are the refractive indices of the waveguiding film, the cover, and the substrate, respectively, $t_{g}$ is the groove depth, and $t_{f}$ is the thickness of the unpatterned film. The grating period along the $z$ direction is denoted by $\Lambda$. Planes $H_{1}$ and $H_{2}$ that are located at $z=z_{1}$ and $z=z_{2}$, respectively, are chosen such that $z_{2}-z_{1}=\Lambda, w$ is the artificial waveguide period (or the computation-window size) in the $x$ direction, $L_{c}$ and $L_{s}$ are the thicknesses of the absorbers for the cover and for the substrate. This kind of grating waveguide has many applications. For example, it can be used as a grating coupler, a Bragg reflector, or a segmented waveguide.

As shown in our previous work, ${ }^{4,5}$ the extension of the RCWA to modelize waveguide diffraction problems relies on an artificial periodization along the $x$ coordinate and on the introduction of absorbers. The artificial periodization along the $x$ coordinate virtually replaces the actual waveguide isolated in space by a periodic waveguide structure. Only one period is shown in Fig. 1. The period (or the computational box size) is denoted by $w$. For the electromagnetic solution of the periodic waveguide geometry and that of the isolated waveguide structures to be identical, absorbers have to be incorporated between the waveguides. These absorbers (represented as rectangles in Fig. 1) are used to satisfy the ingoing wave condition at plane $x=w / 2$ and $x=-w / 2$. As shown in Fig. 1 , the absorbers are composed of two independent parts; one is placed in contact with the cover and the other in contact with the substrate. Note that the absorber permittivity and permeability depend on the refractive indices of the cover and substrate only in an analytical way. As shown in Ref. 5, good absorbers guarantee a good numerical accuracy. We provide results in the following for simple gradient-index and PML absorbers. 


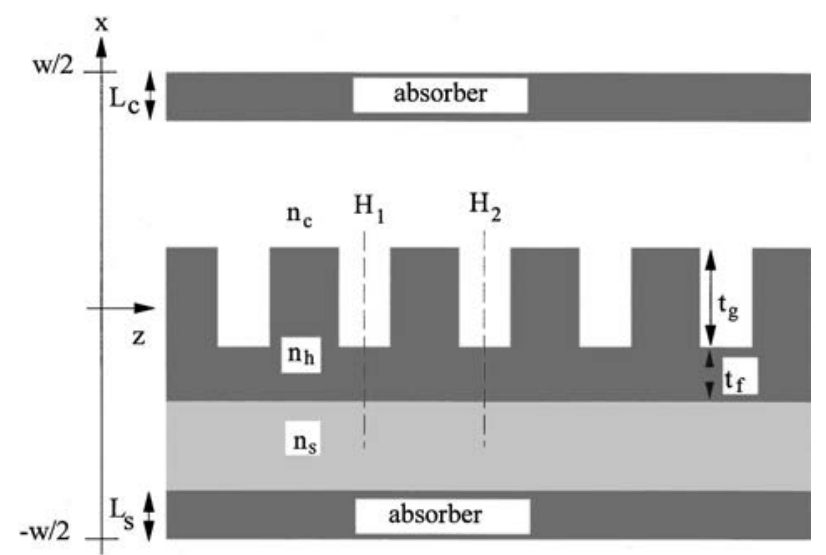

Fig. 1. Grating-waveguide structure considered in this paper. $L_{c}$ and $L_{s}$ are the two absorber thickness, $w$ is the artificial period along the $x$ direction. For the problem of Ref. $11-n_{c}=1$, $n_{h}=\sqrt{3}, n_{s}=\sqrt{2.3}, t_{g}=\lambda, t_{f}=\lambda / \pi$ for $\mathrm{TE}$ waves and $t_{f}$ $=\lambda / 2$ for TM-the groove and ridge lengths are $\lambda / 4(\Lambda=\lambda / 2)$.

With the inclusion of the absorbers that results in a modification of the index-distribution profile, the waveguide modes and their effective indices are computed by solving the Helmoltz equation in every uniform section of the waveguide. For TE polarization, the classical Helmoltz equation is

$$
\frac{\partial^{2} E_{y}}{\partial z^{2}}=-k_{0}^{2} \epsilon E_{y}-\frac{\partial^{2} E_{y}}{\partial x^{2}},
$$

where $E_{y}$ is the $y$ component of the electric field, $k_{0}$ is the modulus of the wave vector and $\epsilon(x)$ is the relative permittivity distribution of the uniform section of the waveguide. A temporal dependence of $\exp (-j \omega t)$ is assumed throughout the paper. When PML absorbers are considered, magnetic and anisotropic media have to be considered and Eq. (1) has to be generalized to ${ }^{5}$

$$
\frac{\partial^{2} E_{y}}{\partial z^{2}}=-k_{0}^{2} \mu_{x x} \epsilon E_{y}-\mu_{x x} \frac{\partial}{\partial x}\left(\frac{1}{\mu_{z z}} \frac{\partial E_{y}}{\partial x}\right),
$$

where $\mu_{x x}(x), \mu_{z z}(x)$, and $\epsilon(x)$ are periodic functions representing the generalized relative permeability and permittivity. They are given by $\mu_{x x}(x)=\mu_{z z}(x)=1$ and $\epsilon(x)=n^{2}(x)$ outside the PML absorbers, and $\mu_{z z}(x)$ $=(1+j) g, \quad \mu_{x x}(x)=1 /[(1+j) g], \quad$ and $\epsilon(x)=n_{c}^{2}(1$ $+j) g$ or $n_{s}^{2}(1+j) g$ inside the PML absorbers. $n(x)$ is the transverse refractive-index distribution of a uniform section of the grating waveguide, and $g$ is a parameter that can be chosen for optimal purpose. The $(1+j)$ factors in the generalized relative permeability and permittivity are introduced to absorb both propagating and evanescent waves in the PML absorbers. ${ }^{5}$ The TMpolarization case can be similarly treated in terms of the symmetric properties $^{5}$ of $\epsilon \leftrightarrow \mu$ and $E \leftrightarrow H$.

Because the structure is periodic in the $x$ coordinate, Eq. (1) or Eq. (2) can be solved with RCWA. The electromagnetic field quantities are first expanded in Fourier series. For the electric field, we have

$$
E_{y}=\sum_{m=-\infty}^{\infty} S_{m}(z) \exp (j m K x),
$$

where $K=2 \pi / w, j^{2}=-1$ and $S_{m}$ is the unknown nor[pmalized amplitude of the $m$ th space-harmonic field. Then Eq. (1) or Eq. (2) is solved as an eigenproblem ${ }^{1,2,5}$ in the Fourier basis to compute the modes in every uniform section of the waveguide. Thus in each section, the electromagnetic field $E_{y}$ is looked for as a superposition of modes

$$
S_{p}(z)=\sum_{m} W_{m}\left\{u_{m} \exp \left[-k_{0} \lambda_{m} z\right]+d_{m} \exp \left[k_{0} \lambda_{m} z\right]\right\},
$$

where $\mathbf{u}$ and $\mathbf{d}$ are column vectors whose elements are the amplitudes of the modes propagating backward (in the $-z$ direction) and forward (in the $z$ direction), respectively. In Eq. (4), $W_{m}$ represents the Fourier coefficient of mode $m$ and $\lambda_{m}$ is the propagation constant of mode $m$.

We denote by $\mathbf{d}_{1}$ and $\mathbf{u}_{1}$ the column vectors whose elements are the amplitudes of the forward- and the backward-propagating modes at plane $H_{1}$. Similarly, $\mathbf{d}_{2}$ and $\mathbf{u}_{2}$ are those corresponding to plane $H_{2}$. For such a grating waveguide, the $\mathbf{T}$ matrix and the $\mathbf{S}$ matrix connect $\mathbf{d}_{1}, \mathbf{u}_{1}, \mathbf{d}_{2}$, and $\mathbf{u}_{2}$ by the following equations, ${ }^{3}$

$$
\begin{aligned}
& \left(\begin{array}{l}
\mathbf{d}_{2} \\
\mathbf{u}_{2}
\end{array}\right)=\mathbf{T}\left(\begin{array}{l}
\mathbf{d}_{1} \\
\mathbf{u}_{1}
\end{array}\right), \\
& \left(\begin{array}{l}
\mathbf{d}_{2} \\
\mathbf{u}_{1}
\end{array}\right)=\left[\begin{array}{l}
\mathbf{S}_{11} \mathbf{S}_{12} \\
\mathbf{S}_{21} \mathbf{S}_{22}
\end{array}\right]\left(\begin{array}{l}
\mathbf{d}_{1} \\
\mathbf{u}_{2}
\end{array}\right),
\end{aligned}
$$

where $\mathbf{T}$ is the $\mathbf{T}$ matrix, $\mathbf{S}_{11}, \mathbf{S}_{12}, \mathbf{S}_{21}$, and $\mathbf{S}_{22}$ are the four submatrices of the $\mathbf{S}$ matrix. Both the $\mathbf{T}$ matrix and the $\mathbf{S}$ matrix can be computed by solving for the eigenproblems associated with the two uniform sections of the grating waveguide and by matching the electromagneticfield quantities at the two interfaces between the two uniform sections. ${ }^{3}$ Since the Bloch mode is a pseudoperiodic function of the $z$ variable, the Bloch-mode column-vector coefficients satisfy $\mathbf{d}_{2}=\beta \mathbf{d}_{1}$ and $\mathbf{u}_{2}=\beta \mathbf{u}_{1}$, where $\beta$ denotes the factor $\exp \left(-i k_{0} n_{\text {eff }} \Lambda\right)$. Substituting these relations into Eqs. (5) and (6) yields the following eigenproblem equations, respectively:

$$
\begin{aligned}
\mathbf{T}\left(\begin{array}{l}
\mathbf{d}_{1} \\
\mathbf{u}_{1}
\end{array}\right) & =\beta\left(\begin{array}{l}
\mathbf{d}_{1} \\
\mathbf{u}_{1}
\end{array}\right), \\
{\left[\begin{array}{rr}
\mathbf{S}_{11} & \mathbf{0} \\
\mathbf{S}_{21} & -\mathbf{1}
\end{array}\right]\left(\begin{array}{l}
\mathbf{d}_{1} \\
\mathbf{u}_{1}
\end{array}\right) } & =\beta\left[\begin{array}{ll}
\mathbf{I} & -\mathbf{S}_{12} \\
\mathbf{0} & -\mathbf{S}_{22}
\end{array}\right]\left(\begin{array}{l}
\mathbf{d}_{1} \\
\mathbf{u}_{1}
\end{array}\right) .
\end{aligned}
$$

In the T-matrix approach of Ref. 4, the eigenvalue $\beta$ and consequently $n_{\text {eff }}$ are directly determined by solving for Eq. (7) as a standard eigenproblem. The method suffers from the well-known numerical instabilities ${ }^{3}$ of the $\mathbf{T}$ matrix. Instead, in the S-matrix approach presented here, the eigenvalues $\beta$ are directly determined by solving for Eq. (8) as a generalized eigenproblem without any instability as evidenced in the following numerical results.

To clearly understand the advantage of the $\mathbf{S}$-matrix approach, we numerically investigate the lamellar grating-waveguide problem studied by Chang et al. ${ }^{11}$ The grating-waveguide parameters are given in the Fig. 1 caption. The $n_{\text {eff }}$ values obtained with the two approaches are shown in Table 1 for TE (electric field parallel to the $y$ direction) and, TM (magnetic field parallel to the $y$ direction) polarizations. For the computation, the 
Table 1. $n_{\text {eff }}$ Values Obtained with the T-Matrix and S-Matrix Approaches ${ }^{a}$

\begin{tabular}{ccc}
\hline$N$ & T Matrix & S Matrix \\
\hline TE Mode $^{b}$ & & \\
21 & $1.578+0.0021 j$ & $1.578+0.0021 j$ \\
41 & $1.582+0.0024 j$ & $1.582+0.0024 j$ \\
61 & $1.583+0.0024 j$ & $1.583+0.0024 j$ \\
81 & $1.583+0.0024 j$ & $1.583+0.0024 j$ \\
161 & $1.579+0.0035 j$ & $1.583+0.0024 j$ \\
301 & - & $1.583+0.0024 j$ \\
TM Mode $^{c}$ & & \\
21 & $1.605+0.00062 j$ & $1.605+0.00062 j$ \\
41 & $1.608+0.00072 j$ & $1.608+0.00072 j$ \\
61 & $1.609+0.00073 j$ & $1.609+0.00073 j$ \\
81 & $1.609+0.00073 j$ & $1.609+0.00073 j$ \\
151 & $1.608+0.00188 j$ & $1.609+0.00074 j$ \\
301 & - & $1.609+0.00074 j$ \\
\hline
\end{tabular}

${ }^{a}$ The absorbers are gradient-index films.

${ }^{b}$ The value obtained in Ref. 8 corresponds to $1.583+0.0022 j$.

${ }^{c}$ The value obtained in Ref. 8 corresponds to $1.609+0.00075 j$.

Table 2. $n_{\text {eff }}$ Values Obtained with the S-Matrix Approach and with Perfectly Matched-Layers

\begin{tabular}{rccc}
\hline$N$ & TE Mode & TM Mode & CPU Times (s) \\
\hline 11 & $1.574+0.0007 j$ & $1.604+0.00102 j$ & 0.1 \\
21 & $1.582+0.0020 j$ & $1.609+0.00078 j$ & 0.3 \\
31 & $1.582+0.0023 j$ & $1.609+0.00065 j$ & 0.5 \\
41 & $1.582+0.0023 j$ & $1.609+0.00071 j$ & 1 \\
61 & $1.582+0.0023 j$ & $1.609+0.00072 j$ & 2.5 \\
301 & $1.582+0.0023 j$ & $1.609+0.00072 j$ & 269 \\
\hline
\end{tabular}

numerical parameters are chosen such that $L_{s}=L_{c}=\lambda$ and $w=6 \lambda$. In this example, the absorbers are composed of gradient-index film stacks with complex refractive-index distributions that vary parabolically from $n_{s}+0 j$ to $n_{s}+1 j$ for the substrate absorber and from $n_{c}+0 j$ to $n_{c}+1 j$ for the cover absorber. Note that the values $\alpha \lambda$ in Table 3 of Ref. 11 is $2 \pi$ times the imaginary parts of $n_{\text {eff }}$. From Table 1, one concludes that the T-matrix approach is unstable for large $N$ values (for example, when $N=301$, no numerical results are obtained). On the other hand, the S-matrix approach is perfectly stable.

As pointed out by Refs. 4 and 5, the absorber quality also has an important influence on the accuracy of the computational results. Good absorbers are required for high performance. Through numerical experiments, we find that the computed effective indices, especially their imaginary parts, are somewhat sensitive to the value of $w$. For example, for TM waves, $n_{\text {eff }}$ converges to 1.609 $+0.00074 j$ for $w=6 \lambda$ but converges to 1.609 $+0.00064 j$ for $w=5 \lambda$; for TE waves, $n_{\text {eff }}$ converges to $1.583+0.0024 j$ for $w=6 \lambda$ but converges to 1.582 $+0.0018 j$ for $w=5 \lambda$. To further improve the accuracy of the approach, we now use PML absorbers. In principle, PML allow us to fulfill exactly the incoming-wave condition in the cover and in the substrate. The effective indices computed with PML are shown in Table 2 for TE and TM polarizations. The corresponding CPU times, which are nearly independent of the polarization, are given in the third column (we use the Matlab version 6.0 and a Pentium III 500-MHz computer). The parameters chosen for the computation are $L_{s}=L_{c}=\lambda / 2, w=3 \lambda$, and $g=5$. A comparative inspection of Tables 1 and 2 clearly evidences the beneficial impact of the PML. In addition, through numerical experiments, we found that the $n_{\text {eff }}$ values are nearly insensitive to the period $w$. Concretely, for $w \geqslant 3 \lambda, n_{\text {eff }}$ always converge to 1.609 $+0.00071 j$ for TM waves and $1.582+0.0023 j$ for TE waves; two values that are in excellent agreement with those of Ref. 11. High accuracy and fast convergence are achieved.

In the above example, the scattered light in the cover is due to the radiation of a single backward harmonic wave. To further test the accuracy of the method, let us now consider the segmented-waveguide structure of Ref. 12 (see Table 1 in Ref. 12 for details concerning the structure), for which more than ten harmonic waves are radiated in the cover and in the substrate. This example allows for a thorough testing of the influence of the absorbers on the accuracy of the method since the Bloch mode strongly radiates and thus interacts with the absorbers. For the computation, the parameters are chosen such that $L_{s}$ $=L_{c}=\lambda, w=7 \mu \mathrm{m}$, and $g=5$. Again we achieve a fast convergence. For example, when 61 Fourier harmonic waves are retained, the computed effective index is $n_{\text {eff }}=1.86026+j 1.333 \times 10^{-6}$ for TM polarization and for a fill factor of 0.5 . This value compares well with the "exact" value $1.8602665+j 1.3409 \times 10^{-6}$ we obtained using the rigorous coupled-wave analysis with 301 Fourier harmonics and searching for the pole of the

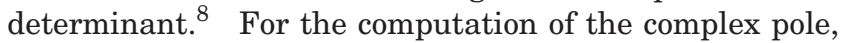
we used a Padé algorithm, and the root search was performed with only a few iterations starting from the initial-guess value provided by the proposed method. Again an excellent agreement is achieved.

The algorithm presented in this communication for the Bloch-mode computation of one-dimensional grating waveguides is unconditionally stable. Although mainly one grating geometry was investigated in this work, we recently applied the method successfully to different grating geometries, such as two-dimensional segmented waveguides, grating couplers, ${ }^{5}$ Bragg gratings, ${ }^{5}$ and photonic-crystal waveguides ${ }^{6}$ and to three-dimensional photonic-crystal waveguides with one line missing. The method provides highly accurate results and a fast convergence rate; and a fourth-digit accuracy was achieved with a CPU time $<1 \mathrm{~s}$ for simple dielectric waveguides such as the one considered in Fig. 1.

\section{ACKNOWLEDGMENTS}

The authors thank Eric Silberstein for fruitful discussions and Pierre Chavel for a critical reading of the manuscript. This work was partly supported under the program Microsystèmes of the Centre National de la Recherche Scientifique. Qing Cao is pleased to acknowledge a fellowship from the Ministère de la Recherche Française. 


\section{REFERENCES}

1. M. G. Moharam, E. B. Grann, D. A. Pommet, and T. K. Gaylord, "Formulation for stable and efficient implementation of the rigorous couple-wave analysis of binary gratings," $\mathrm{J}$. Opt. Soc. Am. A 12, 1068-1076 (1995).

2. Ph. Lalanne and G. M. Morris, "Highly improved convergence of the coupled-wave method for TM polarization," J. Opt. Soc. Am. A 13, 779-784 (1996).

3. L. Li, "Formulation and comparison of two recursive matrix algorithms for modeling layered diffraction gratings," J. Opt. Soc. Am. A 13, 1024-1035 (1996).

4. Ph. Lalanne and E. Silberstein, "Fourier-modal methods applied to waveguide computation problems," Opt. Lett. 25, 1092-1094 (2000).

5. E. Silberstein, P. Lalanne, J. P. Hugonin, and Q. Cao, "Use of grating theories in integrated optics," J. Opt. Soc. Am. A 18, 2865-2875 (2001).

6. M. Palamaru and $\mathrm{Ph}$. Lalanne, "Photonic crystal waveguides: out-of-plane losses and adiabatic modal conversion," Appl. Phys. Lett. 78, 1466-1468 (2001).

7. Ph. Lalanne and H. Benisty, "Out-of-plane losses of twodimensional photonic crystal waveguides: electromagnetic analysis," J. Appl. Phys. 89, 1512-1514 (2001).

8. E. Popov, "Light diffraction by relief gratings: a macroscopic and microscopic view," Prog. Opt. 31, 139-187 (1993).

9. J. P. Berenger, "A perfectly matched layer for the absorption of electromagnetic waves," J. Comput. Phys. 114, 185-200 (1994).

10. C. M. Rappaport, "Interpreting and improving the PML absorbing boundary condition using anisotropic lossy mapping of space," IEEE Trans. Magn. 32, 968-974 (1996).

11. K. C. Chang, V. Shah, and T. Tamir, "Scattering and guiding of waves by dielectric gratings with arbitrary profiles," J. Opt. Soc. Am. 70, 804-812 (1980).

12. L. Li and J. J. Burke, "Linear propagation characteristics of periodically segmented waveguides," Opt. Lett. 17, 11951197 (1992). 